\title{
Nanoscale
}

Check for updates

Cite this: Nanoscale, 2019, 11, 5891

Received 30th November 2018,

Accepted 11th March 2019

DOI: 10.1039/c8nr09671f

rsc.li/nanoscale

\section{Supramolecular chirality and crystallization from biocatalytic self-assembly in lipidic cubic mesophases $\dagger$}

\author{
Tao Zhou, ${ }^{a}$ Jijo J. Vallooran (D) ${ }^{b}$ and Raffaele Mezzenga (D) *a,c
}

\begin{abstract}
Biocatalytic self-assembly in a nanoconfined environment is widely used in nature to construct complex structures that endow special characteristics to life. There is tremendous interest in mimicking such bottom-up processes to fabricate functional materials. In this study, we have investigated a novel biomimetic scaffold based on lipidic cubic mesophases (LCMs), which provide a special nanoconfined environment for biocatalytic self-assembly and subsequent formation of organic crystals. $(R)$-Benzoin generated in situ from benzaldehyde in a reaction catalyzed by the enzyme benzaldehyde lyase (BAL) exhibits - when confined within LCMs - enhanced chirality compared to $(R)$-benzoin in solution or $(R)$-benzoin-doped LCMs. We infer that a metastable state is formed under kinetic control that displays enhanced supramolecular chirality. As they age, these metastable structures can further grow into thermodynamically stable crystals. The biomimetic, nanoconfined environment provided by the LCMs plays a key role in the development of supramolecular chirality and subsequent crystallization.
\end{abstract}

In nature, enzymes are used to control molecular self-assembly processes and thus guide the formation of supramolecular structures, such as basement membranes, which play key roles in biological systems. ${ }^{1,2}$ Most in vivo enzymatic reactions occur in a highly confined environment, such as on a surface or inside a partitioning volume. ${ }^{3-5}$ As a consequence, self-assembly processes in vivo are often controlled by spatial confinement. $^{1-5}$ There is tremendous interest in mimicking such bottom-up processes in order to fabricate functional materials with novel properties and to study biological processes in relevant environments. ${ }^{2,6}$ However, it remains chal-

\footnotetext{
${ }^{a}$ Department of Health Science and Technology, ETH Zurich, Schmelzbergstrasse 9, 8092 Zürich, Switzerland. E-mail: raffaele.mezzenga@hest.ethz.ch

${ }^{b}$ Department of Chemistry, University of Zurich, Winterthurerstrasse 190, 8057 Zurich, Switzerland

${ }^{c}$ Department of Materials, ETH Zurich, Wolfgang-Pauli-Strasse 10, 8093 Zurich, Switzerland

$\dagger$ Electronic supplementary information (ESI) available. See DOI: 10.1039/ c8nr09671f
}

lenging to design systems in which molecules self-assemble spontaneously and exclusively in a highly confined environment. ${ }^{7}$ The most useful approach developed so far to address this problem is nanoconfined biocatalytic self-assembly, such as on the cell surface (2D) or inside the cell (3D), a strategy prevailing in nature. ${ }^{8,9}$ In this approach, precursors, which normally would not undergo self-assembly processes, acquire selfassembling propensities as a consequence of enzymatic triggers. ${ }^{2,10,11}$

Pioneering work in confined biocatalytic self-assembly has been performed by Ulijn et al. and $\mathrm{Xu}$ et al. ${ }^{6,12-18}$ In 2009, Ulijn et al. reported that thermolysin, anchored on a glass surface, can activate the self-assembly of peptides under thermodynamic control. ${ }^{6}$ The confinement created by the glass surface adds an extra control over the self-assembly, thus resulting in fewer defects. ${ }^{6}$ The work by $\mathrm{Xu}$ et al. on novel bottom-up fabrication of nanomaterials shows great promise in biological systems. ${ }^{15-18}$ Enzymes immobilized on the cell surface or in specific subcellular space promote the self-assembly of peptides in the confined environment, which can cause apoptosis in cancer cells. ${ }^{15-18}$ Similar work by Pires et al. showed that membrane-bound alkaline phosphatase (ALP) can induce the formation of a cytotoxic nanonet/hydrogel "cage" surrounding cells and subsequent apoptosis, suggesting that the location of the enzyme has a critical impact on the assembly process. ${ }^{19}$ The aforementioned examples highlight the promise of confined biocatalytic self-assembly for biologically interfacing systems and as a tool for bottom-up nanofabrication. $^{20-24}$

Here, we introduce lipidic cubic mesophases as a medium for biocatalytic self-assembly aiming at controlled design of new nanomaterials. Lipidic cubic mesophases (LCMs), formed by the self-assembly of lipids in water, consist of two sets of interpenetrating, yet noncommunicating water channels, separated by a single, continuous lipid bilayer, with triply periodic surfaces. ${ }^{25}$ Previous work has shown that LCMs are excellent host matrices for enzymatic reactions, due to their biomimetic structures and thermodynamic stability. ${ }^{26-33}$ More interest- 
ingly, the nanoconfined water channels provide a unique environment where enzymatic reactions do not follow Michaelis-Menten kinetics as for the homologue reactions in solution. ${ }^{30}$ In this work, we show that LCMs can also serve as a unique environment for mimicking the confined biocatalytic self-assembly process. We use the enzyme benzaldehyde lyase (BAL) for the biocatalytic self-assembly, incorporating it within LCMs to catalyze the condensation of achiral benzaldehyde to chiral (R)-benzoin (Fig. 1a). BAL has been widely used as a catalyst for asymmetric C-C bond forming reactions. ${ }^{34,35}$ Here, $(R)$-benzoin, produced by BAL in LCMs, shows enhanced chirality compared to both standard $(R)$-benzoin in solution and standard $(R)$-benzoin-doped LCMs. We hypothesize that the enzymatic reaction and nanoconfinement provided by LCMs play key roles in the formation of metastable supramolecular chirality, emerging from the subtle interplay between kinetically trapped states and thermodynamic driving force.

The LCMs in this study consist of $60 \%$ lipid and $40 \%$ buffer solution. Small angle X-ray scattering (SAXS) was used to determine their symmetry. The positions of the Bragg peaks, which follow the sequence $\sqrt{ } 2: \sqrt{ } 3: \sqrt{ } 4: \sqrt{ } 6: \sqrt{ } 8: \sqrt{ } 9$, are a fingerprint of LCMs possessing double-diamond symmetry (Pn3m group), as shown in Fig. 1b. The corresponding lattice parameter is $9.16 \mathrm{~nm}$ (neat cubic phase) and the diameter of the water channels is calculated to be $3.72 \mathrm{~nm}$ (see ESI, section $1.4 \dagger$ and Fig. 1c). In the presence of BAL, a slight increase in both the lattice parameter $(9.35 \mathrm{~nm})$ and the size of water channels $(3.81 \mathrm{~nm})$ was observed, indicative of enzyme incorporation in the cubic lattice. Moreover, the symmetry of the LCM was preserved during the entire biocatalytic

(a)
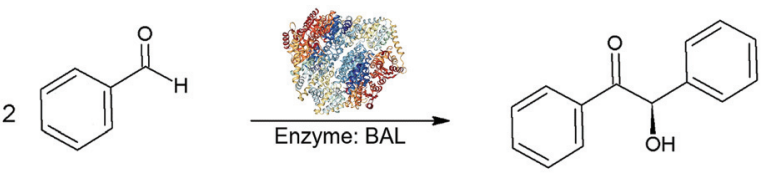

(b)

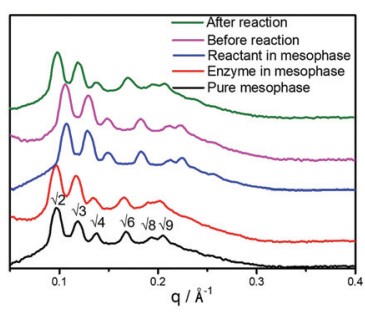

(c)

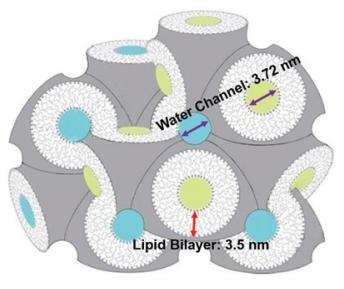

Fig. 1 (a) Formation of chiral $(R)$-benzoin from benzaldehyde catalyzed by the enzyme BAL (PDB code: 2AG0). (b) SAXS spectra of lipidic cubic mesophases (LCMs). Black: pure LCMs prepared by mixing $60 \%$ monolinolein and $40 \%$ buffer solution; red: $1 \mathrm{mg} \mathrm{ml}^{-1}$ enzyme BAL loaded in the LCMs; blue: $0.1 \mathrm{mmol} \mathrm{ml}^{-1}$ reactant benzaldehyde loaded in the LCMs; pink: both enzyme and reactant loaded in the LCM and analysed by SAXS immediately after preparing the sample; green: same as pink, but SAXS analysis was performed after completion of the enzymatic reaction. (c) Schematic of liquid crystals with cubic symmetry Pn3m which constituted the biomimetic lipid bilayer environment used in this work. The two continuous water channels (blue and yellow) which are interpenetrating but noncommunicating to each other separated by a single, continuous lipid bilayer (grey). transformation. HPLC results proved that the high catalytic efficiency of BAL was fully preserved in LCMs (ESI Fig. S1 and $\mathrm{S} 2 \dagger$ ), affording yields and enantiomeric excesses (ee) $>90 \%$.

Apart from being a superior encapsulation medium for enzymes, ${ }^{26-33}$ LCMs also provide special nanoconfined environments for self-assembly of the biocatalytically produced $(R)$-benzoin. The biocatalytic transformations in LCMs were investigated by CD spectroscopy and compared to standard $(R)$-benzoin and $(R)$-benzoin-doped mesophases. Standard $(R)$-benzoin was dissolved in DMSO to obtain the CD information of $(R)$-benzoin (see ESI $\dagger$ ). ${ }^{36}$ The $(R)$-benzoin were fully soluble in DMSO, ${ }^{36}$ which is further certified by quantitative NMR and linearly increased signal of UV, CD and ORD. As shown in Fig. 2a, $(R)$-benzoin has two CD features, a maximum at ca. $290 \mathrm{~nm}$ and a minimum at $317 \mathrm{~nm}$. As expected, the minimum at $317 \mathrm{~nm}$ band increases linearly with increasing concentrations of standard $(R)$-benzoin (obtained from a commercial supplier) (Fig. 2b). Then, we focused on the in situ biocatalytic self-assembly in solution (mixture of $40 \%$ buffer solution and 60\% DMSO) and in LCMs. In solution, no obvious self-assembly was achieved, as there is no CD signal with increasing reaction time (Fig S8a $\dagger$ ). The absence of CD signal results from the spontaneous precipitation of $(R)$-benzoin once converted: indeed, the biocatalytic product, $(R)$-benzoin, precipitated over time in solution and few plate-like crystals were formed (Fig. S8c $\dagger$ ). Most interestingly, however, when the reaction medium changed from solution to LCMs, the converted $(R)$-benzoin displayed a peculiar supramolecular chiral behavior. Need to mention, the reactions carried out in LCMs are characterized by a shift in the CD minimum from $317 \mathrm{~nm}$ to $314 \mathrm{~nm} \cdot{ }^{37,38}$ At low concentrations of substrate (0.02 and $0.06 \mathrm{mmol} \mathrm{ml}^{-1}$ ), this CD signal steadily increased in absolute
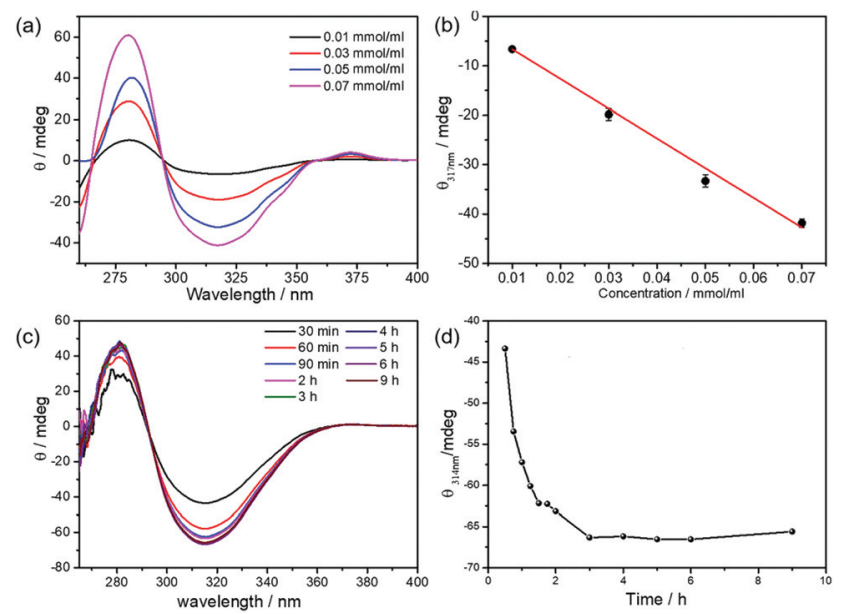

Fig. $2 C D$ measurements of $(R)$-benzoin. (a) Spectra recorded at different concentration of authentic product in solution; (b) linear dependence of the CD signal at $317 \mathrm{~nm}$ as a function of $(R)$-benzoin concentration; (c) CD spectra of $(R)$-benzoin synthesized biocatalytically in situ in LCMs; and (d) evolution of the CD signal at $314 \mathrm{~nm}$ as a function of time. The concentration of substrate in (c) and (d) is $0.14 \mathrm{mmol} \mathrm{ml}^{-1}$. 
value as the reaction progressed before plateauing at a value that remained essentially constant over time (Fig. S9†). The final signal was nearly the same as that observed for the same concentration of authentic $(R)$-benzoin in solution. Interestingly, however, when the substrate concentration was increased to $0.10 \mathrm{mmol} \mathrm{ml}^{-1}$, the CD signal increased in absolute value steadily over three hours to a final value of $-50 \mathrm{mdeg}$ (Fig. S10†), considerably enhanced over the value of -30 mdeg expected for quantitative conversion to $(R)$-benzoin in solution. Similar behavior was also observed at higher substrate concentrations: for reactions carried out with $0.14 \mathrm{mmol} \mathrm{ml}^{-1}$ benzaldehyde, the CD signal reached -66 mdeg in LCMs versus -41 mdeg for the solution control, corresponding to a $60 \%$ enhanced CD signal change (Fig. 2c and d). These results clearly show that benzoin condensations carried out in LCMs lead to enhanced chirality compared to their solution counterparts.

As a next step, we studied the CD signal of LCMs doped with $(R)$-benzoin from a commercial supplier in order to check whether the enhanced chirality observed for the LCM samples results from mere accumulation of product in LCMs. For this purpose, we doped LCMs with different concentrations of $(R)$ benzoin and compared the CD spectra of the resulting solutions (Fig. S11 $\dagger$ ) with those for the same concentrations of $(R)$ benzoin synthesized in situ in the LCMs. In the low concentration range, doped LCMs exhibited the same CD signal as enzymatically synthesized $(R)$-benzoin. However, when the concentration was increased, doped LCMs exhibited very low signals, even lower than solutions of authentic sample, likely due to precipitation of the molecule. This result supports the conclusion that enhanced chirality is unique to the enzymatic reactions that generate $(R)$-benzoin in situ, and does not arise from simple accumulation of product in the partitioning compartments of the LCMs.

Fig. 3a summarizes the $\mathrm{CD}$ results, highlighting the enhanced chirality of $(R)$-benzoin prepared in LCMs at concentrations of $0.05 \mathrm{mmol} \mathrm{ml} \mathrm{m}^{-1}$ or higher. The enhanced chirality was confirmed by independent optical rotatory dispersion (ORD) measurements. As shown in Fig. 3b, the ORD signal of free $(R)$-benzoin in solution increases linearly with increasing concentration. For low concentrations, such as $0.01 \mathrm{mmol}$ $\mathrm{ml}^{-1}$ and $0.03 \mathrm{mmol} \mathrm{ml}^{-1},(R)$-benzoin synthesized in LCMs has the same ORD signal as free $(R)$-benzoin in solution. However, just as for the CD spectra, if the concentration is

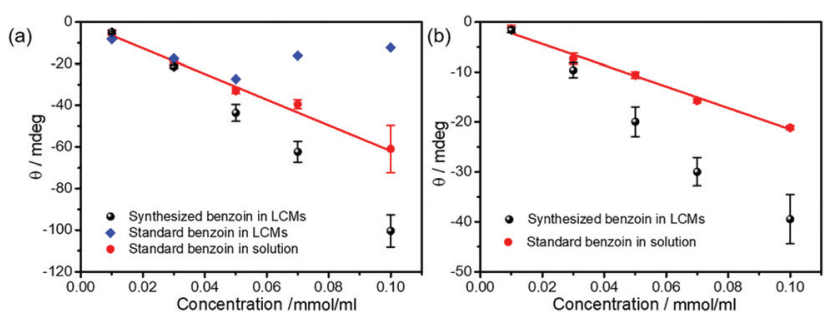

Fig. 3 (a) $C D$ and (b) ORD measurements of $(R)$-benzoin from a commercial supplier and $(R)$-benzoin produced biocatalytically in solution (red) and in LCMs (black). increased to $0.05 \mathrm{mmol} \mathrm{ml} \mathrm{m}^{-1}$ and beyond, the $(R)$-benzoin produced in LCMs reaches a significantly higher ORD signal, again demonstrating enhanced chirality. Taking together the results from CD and ORD analysis, it is clear that supramolecular chirality is successfully established via biocatalytic selfassembly in LCMs.

Another interesting phenomenon is observed when the concentration of substrate is increased to $0.2 \mathrm{mmol} \mathrm{ml}^{-1}$. As seen in Fig. 4a and b, enhanced chirality is achieved very rapidly, that is within the first 60-90 min. The CD signal of $0.1 \mathrm{mmol}$ $\mathrm{ml}^{-1}(R)$-benzoin in solution is only $-60 \mathrm{mdeg}$ while the CD signal in LCM amplifies to -106 mdeg by 2 hours and then remained constant till 5 hours. However, afterwards, the CD signal starts to fade slowly, reaching a value of -25 mdeg after 24 hours (Fig. 4a). This drastic decrease in CD signal intensity is due to the formation of crystals after 24 hours (Fig. $4 \mathrm{~d}$ ). The crystals grown from the standard $(R)$-benzoin dissolved in a solvent show negligible CD signal (Fig. S12†). Interestingly, the final CD signal is very similar to the CD signal of doped $(R)$ benzoin in LCMs. Conversely, samples at lower concentrations of $(R)$-benzoin for which no crystals are formed, maintain a monotonic evolution of CD intensity (Fig. 4b), confirming that the fading of the CD signal is associated with the crystal formation. Additionally, real-time HPLC results confirmed that synthesized $(R)$-benzoin was not converted to benzaldehyde (Fig. 4c). The HPLC results also show that the reaction is almost complete after 8 hours, while there is no sign of crystals until 24 hours. Taken together, these results indicate that the product is kinetically trapped in a supramolecular chiral state and then relaxes over time to afford thermodynamically favored crystals. It is widely known that crystals are the most stable species. $^{39-41}$ It has been reported previously that kinetically controlled superstructures, such as fibers, can transform into more thermodynamically stable crystals upon aging. ${ }^{42-44}$ Here,
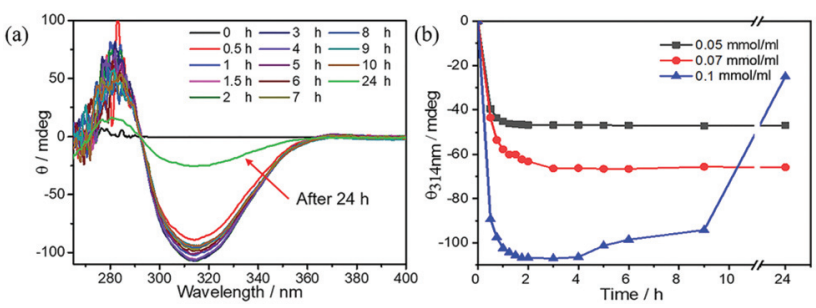

(c)
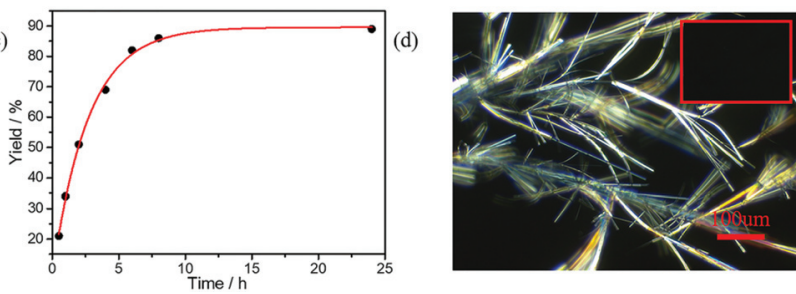

Fig. 4 (a) $C D$ spectra and (b) in time $C D$ measurement of synthesized $(R)$-benzoin in LCMs. (c) In time HPLC measurement of biocatalytic $(R)$ benzoin in LCMs. (d) Polarized optical microscopy (POM) images of synthesized $(R)$-benzoin crystals in LCMs after $24 \mathrm{~h}$, the inset POM image is obtained after $9 \mathrm{~h}$. The concentration of substrate is $0.2 \mathrm{mmol} \mathrm{ml}^{-1}$. 


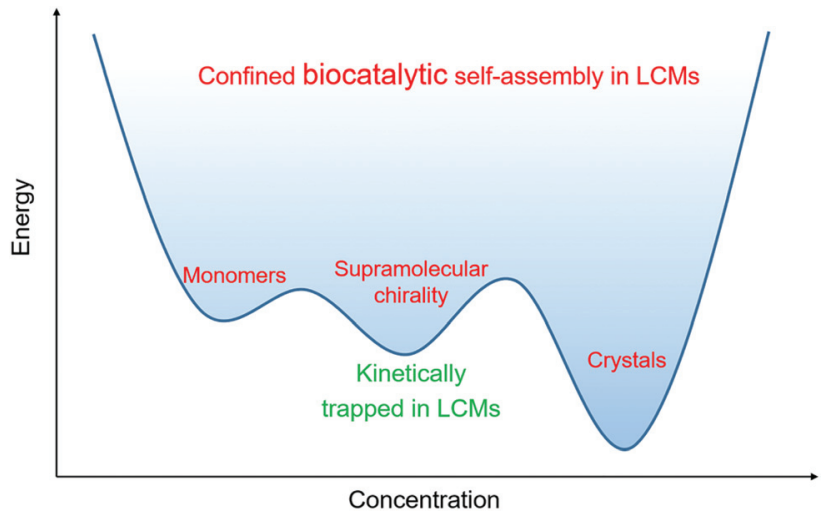

Fig. 5 Suggested schematic of biocatalytic self-assembly and crystallization in LCMs under kinetic and thermodynamic control.

we presume that at intermediate product concentrations a metastable state is formed that shows enhanced supramolecular chirality and is under kinetic control. These metastable structures can further evolve into the thermodynamically favored state (crystals), as shown in Fig. 5. Upon further increase in concentration, the thermodynamically favored crystals are formed rapidly. After only 30 minutes, a large amount of crystals can be observed via polarized optical microscopy (POM) (Fig S13-15†). Interestingly, all the LCMs in which crystals formed show very similar CD spectra (Fig. S16†), likely because some soluble $(R)$-benzoin remains trapped in the channels. Pure crystals formed from standard $(R)$-benzoin dissolved in a volatile solvent did not exhibit a CD signal (Fig. S12†).

\section{Conclusions}

In this work, we successfully mimicked a spatially confined biocatalytic self-assembly process in LCMs. The CD and ORD results show that $(R)$-benzoin generated by BAL in LCMs exhibits enhanced chirality compared to $(R)$-benzoin in solution or added exogenously to LCMs. These data recapitulate key roles of spatially confined environments on enzymatic conversions, leading to the formation of supramolecular chirality. When these structures age, they are gradually transformed into thermodynamically favored crystals. Overall, this special bottom-up material fabrication in a biomimetic environment should give chemists additional tools to create more complex and functional materials.

\section{Conflicts of interest}

There are no conflicts to declare.

\section{Acknowledgements}

The authors acknowledge Donald Hilvert and Raphael Frey for enzyme BAL. The authors acknowledge Ortner Franziska and
Huber Markus for chiral HPLC measurement. Tao Zhou acknowledges the China Scholarship Council and ETH Zurich for financial support of this work.

\section{References}

1 H. Lodish, A. Berk, P. Matsudaira, C. A. Kaiser, M. Krieger, M. P. Scott, S. L. Zipursky and J. Darnell, Molecular Cell Biology, Freeman, New York, 5th edn, 2003.

2 Z. M. Yang, G. L. Liang and B. Xu, Acc. Chem. Res., 2008, 41, 315-326.

3 A. Kuchler, M. Yoshimoto, S. Luginbuhl, F. Mavelli and P. Walde, Nat. Nanotechnol., 2016, 11, 409-420.

4 B. Alberts, A. Johnson, J. Lewis, M. Raff, K. Roberts and P. Walter, Molecular Biology of the Cell, Garland Science, New York, 2002.

5 R. J. Conrado, J. D. Varner and M. P. Delisa, Curr. Opin. Biotechnol., 2008, 19, 492-499.

6 R. J. Williams, A. M. Smith, R. Collins, N. Hodson, A. K. Das and R. V. Ulijn, Nat. Nanotechnol., 2009, 4, 19-24.

7 C. Vigier-Carrière, T. Garnier, D. Wagner, P. Lavalle, M. Rabineau, J. Hemmerlé, B. Senger, P. Schaaf, F. Boulmedais and L. Jierry, Angew. Chem., Int. Ed., 2015, 54, 10198-10201.

8 C. Vigier-Carrière, D. Wagner, A. Chaumont, B. Durr, P. Lavalle, C. Lambour, M. Schmutz, J. Hemmerlé, B. Senger, B. Senger, P. Schaaf, F. Boulmedais and L. Jierry, Langmuir, 2017, 33, 8267-8276.

9 A. R. Hirst, S. Roy, M. Arora, A. K. Das, N. Hodson, P. Murray, S. Marshall, N. Javid, J. Sefcik, J. Boekhoven, J. H. V. Esch, S. Santabarbara, N. T. Hunt and R. V. Ulijn, Nat. Chem., 2010, 2, 1089-1094.

10 S. H. Um, J. B. Lee, N. Park, S. Y. Kwon, C. C. Umbach and D. Luo, Nat. Mater., 2006, 5, 797-801.

11 Y. Liu, H. C. Wu, N. Bhokisham, J. Y. Li, K. L. Hong, D. N. Quan, C. Y. Tsao, W. E. Bentley and G. F. Payne, Bioconjugate Chem., 2018, 29, 1809-1822.

12 M. P. Conte, K. H. A. Lau and R. V. Ulijn, ACS Appl. Mater. Interfaces, 2017, 9, 3266-3271.

13 M. P. Conte, J. K. Sahoo, Y. M. Abul-Haija, K. H. A. Lau and R. V. Ulijn, ACS Appl. Mater. Interfaces, 2018, 10, 3069-3075.

14 D. Kalafatovic, M. Nobis, J. Son, K. I. Anderson and R. V. Ulijn, Biomaterials, 2016, 98, 192-202.

15 Z. Q. Q. Feng, H. M. Wang, R. Zhou, J. Li and B. Xu, J. Am. Chem. Soc., 2017, 139, 3950-3953.

16 H. M. Wang, Z. Q. Q. Feng, D. D. Wu, K. J. Fritzsching, M. Rigney, J. Zhou, Y. J. Jiang, K. Schmidt-Rohr and B. Xu, J. Am. Chem. Soc., 2016, 138, 10758-10761.

17 Y. Kuang, J. Shi, J. Li, D. Yuan, K. A. Alberti, Q. Xu and B. Xu, Angew. Chem., Int. Ed., 2014, 53, 8104.

18 J. Zhou, X. Du, C. Berciu, H. He, J. Shi, D. Nicastro and B. Xu, Chem., 2016, 1, 246-263.

19 R. A. Pires, Y. M. Abul-Haija, D. S. Costa, R. NovoaCarballal, R. L. Reis, R. V. Ulijn and I. Pashkuleva, J. Am. Chem. Soc., 2015, 137, 576-579. 
20 Z. M. Yang, G. L. Liang, Z. F. Guo, Z. H. Guo and B. Xu, Angew. Chem., Int. Ed., 2007, 46, 8216-8219.

21 Y. Kuang and B. Xu, Angew. Chem., Int. Ed., 2013, 52, 69446948.

22 J. Zhou, X. W. Du, J. Li, N. Yamagata and B. Xu, J. Am. Chem. Soc., 2015, 137, 10040-10043.

23 Y. Gao, J. F. Shi, D. Yuan and B. Xu, Nat. Commun., 2012, 3, 1033.

24 J. Li, J. F. Shi, J. E. Medina, J. Zhou, X. W. Du, H. M. Wang, C. H. Yang, J. F. Liu, Z. M. Yang, D. M. Dinulescu and B. Xu, Adv. Healthcare Mater., 2017, 6, 1601400.

25 N. Garti, P. Somasndaran and R. Mezzenga, Self-assembled Supermolecular Architectures: Lyotropic Liquid Crystals, A John Wiley \& Sons Inc., New York, America, 2012, pp. 1-20.

26 D. F. Li and M. Caffrey, Proc. Natl. Acad. Sci. U. S. A., 2011, 108, 8639-8644.

27 E. Nazaruk, E. Gorecka and R. Bilewicz, J. Colloid Interface Sci., 2012, 385, 130-136.

28 W. K. Fong, T. Hanley and B. J. Boyd, J. Controlled Release, 2009, 135, 218-226.

29 T. Zhou, J. J. Vallooran, S. Assenza, A. Szekrenyi, P. Clapés and R. Mezzenga, ACS Catal., 2018, 7, 58105815.

30 J. J. Vallooran, S. Handschin, S. M. Pillai, B. N. Vetter, S. Rusch, H.-P. Beck and R. Mezzenga, Adv. Funct. Mater., 2016, 26, 181-190.

31 W. J. Sun, J. J. Vallooran, A. Zabara and R. Mezzenga, Nanoscale, 2014, 6, 6853-6859.
32 W. J. Sun, J. J. Vallooran and R. Mezzenga, Langmuir, 2015, 31, 4558-4565.

33 W. J. Sun, J. J. Vallooran, W. K. Fong and R. Mezzenga, J. Phys. Chem. Lett., 2016, 7, 1507-1512.

34 A. S. Demir, Ö. Sesenoglu, E. Eren, B. Hosrik, M. Pohl, E. Janzen, D. Kolter, R. Feldmann, P. Dünkelmann and M. Müller, Adv. Synth. Catal., 2002, 344, 96-103.

35 S. Shanmuganathan, D. Natalia, A. V. D. Wittenboer, C. Kohlmann, L. Greiner and P. D. D. Maria, Green Chem., 2010, 12, 2240-2245.

36 L. H. Keith and D. B. Walters, National Toxicology Program's Chemical Solubility Compendium, Taylor and Francis, 1991, p. 51.

37 J. H. Bredehöft, N. Jones, C. Meinert, A. Evens, S. Hoffmann and U. Meierhenrich, Chirality, 2014, 26, 373-378.

38 F. Zsila, Z. Bikàdi and M. Simonyi, Org. Biomol. Chem., 2004, 2, 2902-2910.

39 D. J. Adams, K. Morris, L. Chen, L. C. Serpell, J. Bacsa and G. M. Day, Soft Matter, 2010, 6, 4144-4156.

40 S. Mondal, P. Chakraborty, S. Das, P. Bairi and A. K. Nandi, Langmuir, 2016, 32, 5373-5382.

41 J. Wang, K. Liu, R. R. Xing and X. H. Yan, Chem. Soc. Rev., 2016, 45, 5589-5604.

42 P. L. Zhu, X. H. Yan, Y. Su, Y. Yang and J. B. Li, Chem. Eur. J., 2010, 16, 3176-3183.

43 Y. J. Wang, L. M. Tang and J. Yu, Cryst. Growth Des., 2008, 8, 884-889.

44 J. Adamcik and R. Mezzenga, Angew. Chem., Int. Ed., 2018, 57, 8370-8382. 